TRANSACTIONS OF THE

AMERICAN MATHEMATICAL SOCIETY

Volume 357, Number 2, Pages 489-507

S 0002-9947(04)03474- 9

Article electronically published on March 23, 2004

\title{
MODULI OF SUSPENSION SPECTRA
}

\author{
JOHN R. KLEIN
}

\begin{abstract}
For a 1-connected spectrum $E$, we study the moduli space of suspension spectra which come equipped with a weak equivalence to $E$. We construct a spectral sequence converging to the homotopy of the moduli space in positive degrees. In the metastable range, we get a complete homotopical classification of the path components of the moduli space. Our main tool is Goodwillie's calculus of homotopy functors.
\end{abstract}

\section{INTRODUCTION}

A well-known property of the singular cohomology of a space is that it possesses the structure of a commutative graded ring. There is no corresponding property for the singular cohomology of spectra. This discrepancy between spaces and spectra is connected with the observation that a spectrum $E$ can fail to have a "diagonal map" $E \rightarrow E \wedge E$. Of course, a diagonal map exists when $E$ is a suspension spectrum. This motivates the following:

Question. Let $E$ be a spectrum. When can we find a based space $X$ and a weak equivalence of spectra $\Sigma^{\infty} X \simeq E$ ? In how many ways?

The first result of this paper gives a criterion for deciding the existence part of the above question in the metastable range. Recall that $E$ is $r$-connected if its homotopy groups $\pi_{*}(E)$ vanish when $* \leq r$. Write $\operatorname{dim} E \leq n$ if $E$ is, up to homotopy, a CW spectrum with cells in dimensions $\leq n$. Recall that the $k$-th extended power $D_{k}(E)$ is the homotopy orbit spectrum of the symmetric group $\Sigma_{k}$ acting on the $k$-fold smash product $E^{\wedge k}$.

Theorem A (Existence). There is an obstruction

$$
\delta_{E} \in\left[E, \Sigma D_{2}(E)\right]
$$

which is trivial when $E$ has the homotopy type of a suspension spectrum.

Conversely, if $\delta_{E}=0, E$ is $r$-connected, $r \geq 1$ and $\operatorname{dim} E \leq 3 r+2$, then $E$ has the homotopy type of a suspension spectrum.

Before stating our second result, we comment on the relation between Theorem $\AA$ and one of the early results of Kuhn ( $\mathrm{Ku}$, Th. 1.2]) which says that a connected spectrum $E$ is a retract of a suspension spectrum if and only if there is a weak equivalence

$$
\Sigma^{\infty} \Omega^{\infty} E \simeq \bigvee_{k \geq 1} D_{k}(E)
$$

Received by the editors January 3, 2003 and, in revised form, July 1, 2003.

2000 Mathematics Subject Classification. Primary 55P42, 55P43; Secondary 55P40, 55P65.

The author was partially supported by NSF Grant DMS-0201695. 
Theorem A implies one can remove the word "retract" from Kuhn's result in the metastable range.

Next consider the collection of pairs $(X, h)$ in which $X$ is a based space and $h: \Sigma^{\infty} X \rightarrow E$ is a weak (homotopy) equivalence. Equate two such pairs $(X, h)$ and $(Y, g)$ if and only if there is a map of spaces $f: X \rightarrow Y$ such that $g \circ \Sigma^{\infty} f$ is homotopic to $h$ (in particular, $f$ is a homology isomorphism). This generates an equivalence relation. Let $\Theta_{E}$ denote the associated set of equivalence classes.

Our second result identifies $\Theta_{E}$ in the metastable range.

Theorem B (Enumeration). Assume $\Theta_{E}$ is nonempty and is equipped with basepoint. Then there is a basepoint preserving function

$$
\phi: \Theta_{E} \rightarrow\left[E, D_{2}(E)\right] .
$$

If $E$ is $r$-connected, $r \geq 1$ and $\operatorname{dim} E \leq 3 r+2$, then $\phi$ is a surjection. If, in addition, $\operatorname{dim} E \leq 3 r+1$, then $\phi$ is a bijection. In particular, $\Theta_{E}$ has the structure of an abelian group when $\operatorname{dim} E \leq 3 r+1$.

Theorem Bleads to the possibility of calculating $\Theta_{E}$ in a number of simple cases. For example, Table 4.1 appearing in Mahowald's memoir [Mah] provides extensive calculations of $\Theta_{E}$ at the prime 2 in the case of two cell spectra (see $\S 8$ below).

In fact, $\Theta_{E}$ is the set of path components of a space $\mathfrak{M}_{E}$ which is defined as follows: let $C_{E}$ be the category whose objects are pairs $(Y, h)$ with $Y$ a based space and $h: \Sigma^{\infty} Y \rightarrow E$ a weak equivalence. A morphism $(Y, h) \rightarrow(Z, g)$ consists of a map of based spaces $f: Y \rightarrow Z$ such that $g \circ \Sigma^{\infty} f=h$. Then define

$$
\mathfrak{M}_{E}:=\left|C_{E}\right|,
$$

i.e., the geometric realization of (the nerve of) $C_{E}$.1 We call $\mathfrak{M}_{E}$ the moduli space of suspension structures on $E$.

Our third result gives a spectral sequence converging to the homotopy of $\mathfrak{M}_{E}$ in positive degrees. Formulating it requires some preparation.

For $q \geq 2$, let $W_{q}$ be the spectrum with $\Sigma_{q}$-action that classifies the $q$-th layer of the Goodwillie tower of the identity functor from based spaces to based spaces. Unequivariantly, $W_{q}$ has the homotopy type of a wedge of $(q-1)$ ! copies of the $(1-q)$-sphere spectrum (see Johnson [Jo, Arone [Ar]). If $q \leq 1$, we take $W_{q}$ to be the trivial spectrum.

If $E$ is a spectrum, then its $q$-fold smash power $E^{\wedge q}$ has the structure of a spectrum with (naive) $\Sigma_{q}$-action. Give the smash product $W_{q} \wedge E^{\wedge q}$ the diagonal $\Sigma_{q}$-action. We are then entitled to form

$$
W_{q} \wedge_{h \Sigma_{q}} E^{\wedge q},
$$

i.e, the homotopy orbit spectrum of $\Sigma_{q}$ acting on $W_{q} \wedge E^{\wedge q}$. Let $F\left(E, W_{q} \wedge_{h \Sigma_{q}} E^{\wedge q}\right)$ denote the function space of spectrum maps from $E$ to $W_{q} \wedge_{h \Sigma_{q}} E^{\wedge q}$. With these definitions we are ready to state our third result.

Theorem C (Spectral Sequence). Let $E$ be 1-connected. Assume $\mathfrak{M}_{E}$ is nonempty and equipped with basepoint. Then there is a first quadrant spectral sequence

\footnotetext{
${ }^{1}$ There are set-theoretic difficulties presented by this definition, since $C_{E}$ isn't a small category. The problem can be avoided in a variety of ways, all furnishing the same homotopy type (compare Wa p. 379]).
} 
converging to $\pi_{p+1}\left(\mathfrak{M}_{E}\right)$ with

$$
E_{p, q}^{1}:=\pi_{p}\left(F\left(E, W_{q} \wedge_{h \Sigma_{q}} E^{\wedge q}\right)\right)
$$

and $d^{1}$-differential of bi-degree $(-1,1)$.

Note when $\operatorname{dim} E \leq n$, we have $E_{p, q}^{1}=0$ for $p \leq q+1-n$. Thus for fixed $p, E_{p, q}^{1}$ is nonzero for finitely many $q$.

Our last result says that the obstruction $\delta_{E}$ is 2-local.

Theorem D (Localization). For any connected spectrum E, the homotopy class $\delta_{E}$ becomes trivial after inverting the prime 2.

Furthermore, if $f: E \rightarrow E^{\prime}$ is a map of spectra which is a 2-local weak equivalence, then $\delta_{E}=0$ if and only if $\delta_{E^{\prime}}=0$.

Consequently, if $E$ and $E^{\prime}$ satisfy the connectivity assumptions of Theorem $A$, then $E$ is weak equivalent to a suspension spectrum if and only if $E^{\prime}$ is weak equivalent to a suspension spectrum.

Remarks. Since the paper of Berstein and Hilton $\mathrm{B}-\mathrm{H}]$, the problem of deciding when a space has the homotopy type of a (single or iterated) suspension has been intensively studied.

To the best of my knowledge, the literature contains much less information about the desuspension problem for spectra, aside from the trivial stable range case (Freudenthal's theorem) and a $p$-local version considered by Gray [Gr.

My original interest in the desuspension question for spectra came from embedding theory. In a future paper, we intend to use the above results to attack certain embedding questions.

Outline. $\S 2$ consists of preliminary material. The proof of Theorem $\mathrm{A}$ is contained in $\S 3$. In $\S 4$ we prove Theorem B In $\S 5$ we express the connected components of the moduli space $\mathfrak{M}_{E}$ as the classifying space of a suitable monoid. $\S 6$ contains the proof of Theorem $\mathrm{C}$ Theorem $\mathrm{D}$ is proved in $\S 7$. In $\S 8$ we provide examples in connection with Theorem B In $\S 9$ we discuss some loose ends. $\S 10$, which should be of independent interest, outlines the classification of quadratic homotopy functors from spectra to spectra. The classification presented here is due to Goodwillie, but I first learned about a special case of it from Kuhn. The classification gives a more concrete description of the invariant $\delta_{E}$.

\section{Preliminaries}

In this section we give the conventions and tools used throughout the paper.

Spaces. All spaces will be compactly generated, and Top will denote the category of compactly generated spaces. In particular, we make the convention that products are to be retopologized with respect to the compactly generated topology. Let $\mathbf{T o p}_{*}$ denote the category of based spaces. A weak equivalence of spaces is shorthand for (a chain of) weak homotopy equivalence(s).

We use the usual connectivity terminology for spaces: A nonempty space is $r$ connected if its homotopy vanishes in degrees $\leq r$ with respect to any choice of basepoint (note: every nonempty space is (-1)-connected). A map $A \rightarrow B$ of spaces, with $B$ nonempty, is $r$-connected if for any choice of basepoint in $B$, the homotopy fiber with respect to this choice of basepoint is an $(r-1)$-connected space. 
A commutative square of spaces

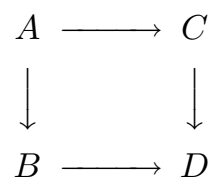

is said to be $k$-cocartesian if the map $\operatorname{hocolim}(B \leftarrow A \rightarrow C) \rightarrow D$ is $k$-connected. Similarly, the square is $k$-cartesian if the map $A \rightarrow \operatorname{holim}(B \rightarrow D \leftarrow C)$ is $k$ connected. The square is $\infty$-(co)cartesian if it is $k-($ co)cartesian for every $k$.

Spectra. A spectrum $E$ will be taken to mean a collection of based spaces $\left\{E_{i}\right\}_{i \in \mathbb{N}}$ together with based (structure) maps $\Sigma E_{i} \rightarrow E_{i+1}$ where $\Sigma E_{i}$ is the reduced suspension of $E_{i}$. A morphism of spectra $E \rightarrow E^{\prime}$ consists of maps $E_{i} \rightarrow E_{i}^{\prime}$ that are compatible with the structure maps. We denote the category of spectra by $\mathbf{S p}$.

A map of spectra is $r$-connected if it induces a surjection on homotopy up through degree $r$ and an isomorphism in degrees less than $r$. A spectrum is $r$-connected if the map to the zero object (consisting of the one point space in each degree) is $(r+1)$-connected.

A map of spectra is a weak equivalence if it is $r$-connected for all integers $r$. This notion of weak equivalence comes from a Quillen model structure on Sp. In this model structure, the fibrant objects are the $\Omega$-spectra (those spectra $E$ such that $E_{i} \rightarrow \Omega E_{i+1}$ is a weak equivalence for all $i$. A cofibrant object is (a retract of) a spectrum which is built up from the zero object by attaching cells. The model structure on $\mathbf{S p}$ comes equipped with functorial factorizations; in particular, fibrant and cofibrant approximation are functorial. For details, see Schwede $\underline{\mathrm{Sc}}$.

We typically apply fibrant and/or cofibrant approximation functors to maintain homotopy invariance. To avoid clutter, we usually suppress the application of these approximations in the notation.

For example, if $E$ is a fibrant spectrum, we write $\Omega^{\infty} E$ for the zero space $E_{0}$ of $E$. If $E$ isn't fibrant, to get a good construction we first replace $E$ by its associated $\Omega$-spectrum $E^{\sharp}$ and then define $\Omega^{\infty} E$ as $E_{0}^{\sharp}$.

If $X$ is a based space, its suspension spectrum $\Sigma^{\infty} X$ has $j$-th space $\Sigma^{j} X$, the $j$-fold reduced suspension of $X$ (for this to have the correct homotopy type, we assume that $X$ is a cofibrant space; i.e., the retract of a cell complex). In particular, $Q(X):=\Omega^{\infty} \Sigma^{\infty} X$ is the reduced stable homotopy functor. A map of spaces $Y \rightarrow \Omega^{\infty} E$ is adjoint to a map of spectra $\Sigma^{\infty} Y \rightarrow E$.

The notion of $r$-cartesian and $r$-cocartesian squares generalizes to spectra in the obvious way. Note that an $r$-cartesian square is the same thing as an $(r+1)$ cocartesian square in the category of spectra. A commutative diagram such as (1) but this time of spectra, which is $\infty$-cartesian and with $C$ weak equivalent to the zero object, is called a fibration sequence (for spectra, a fibration sequence is the same thing as a cofibration sequence). By slight abuse of notation, we write the sequence as $A \rightarrow B \rightarrow D$.

As in the introduction, we write $\operatorname{dim} E \leq n$ if and only if $E$ is, up to homotopy, obtained from the zero object by attaching cells of dimension $\leq n$. A spectrum is finite if it is built up from the zero object by attaching a finite number of cells. A spectrum is homotopy finite if it is weak equivalent to a finite spectrum.

In this paper, we can get away with a notion of smash product which is associative, commutative and unital up to homotopy (see e.g. Lewis, May and Steinberger 
[L-M-S, Chap. 2]). We also need to know that the extended power spectrum

$$
D_{k}(E)=\left(E^{\wedge k}\right)_{h \Sigma_{k}}:=\left(E \Sigma_{k}\right)_{+} \wedge_{\Sigma_{k}} E^{\wedge k}
$$

exists, is functorial, homotopy invariant and coincides with the usual one in the case of spaces. For details, see [L-M-S, Chap. 6].

Truncation. Let $Y$ be a based space, $W$ a 1-connected spectrum and $f: \Sigma^{\infty} Y \rightarrow$ $W$ a map of spectra. Assume $\operatorname{dim} W \leq n$ and that $f$ is $n$-connected.

Lemma 2.1. If $n \geq 0$, there exists a space $Z$, and an $(n-1)$-connected map $g: Z \rightarrow$ $Y$ such that the composite

$$
\Sigma^{\infty} Z \stackrel{\Sigma^{\infty} g}{\rightarrow} \Sigma^{\infty} Y \stackrel{f}{\rightarrow} W
$$

is a weak equivalence.

Proof. If $n \leq 1$, then $W$ is weak equivalent to the zero object, and the proof is trivial in this case.

Assume then that $n \geq 2$. Let $h \mathbb{Z}$ be the Eilenberg-Mac Lane spectrum. Then the induced map

$$
h \mathbb{Z} \wedge \Sigma^{\infty} Y \rightarrow h \mathbb{Z} \wedge W
$$

can be thought of as a map of chain complexes (via the Dold-Kan correspondence). Applying the truncation lemma [Kl1, 4.1], We obtain a space $Z$ and an (n-1)connected map $Z \rightarrow Y$ such that the composite

$$
h \mathbb{Z} \wedge \Sigma^{\infty} Z \rightarrow h \mathbb{Z} \wedge \Sigma^{\infty} Y \rightarrow h \mathbb{Z} \wedge W
$$

is a weak equivalence. But this composite is obtained by smashing the composite

$$
\Sigma^{\infty} Z \rightarrow \Sigma^{\infty} Y \rightarrow W
$$

with $h \mathbb{Z}$. The result then follows by Whitehead's theorem.

Corollary 2.2 (Freudenthal). Let $E$ be a an $r$-connected spectrum, $r \geq 1$. Assume $\operatorname{dim} E \leq 2 r+2$. Then $E$ is weak equivalent to a suspension spectrum.

Proof. This follows from 2.1 because the map $\Sigma^{\infty} \Omega^{\infty} E \rightarrow E$ is $(2 r+2)$-connected (see 3.2 below).

Another description of the moduli space. Let $C_{E}^{\prime}$ be the (not full) subcategory of $C_{E}$ having the same objects, and whose morphisms $(Y, h) \rightarrow\left(Y^{\prime}, h^{\prime}\right)$ satisfy the condition that $Y \rightarrow Y^{\prime}$ is a weak homotopy equivalence. Let $\mathfrak{M}_{E}^{\prime}$ denote the realization of $C_{E}^{\prime}$.

Proposition 2.3. Assume that $E$ is fibrant, cofibrant and 1-connected. Then the inclusion $\mathfrak{M}_{E}^{\prime} \subset \mathfrak{M}_{E}$ is a homotopy equivalence.

Proof. Let $Y \mapsto Y^{+}$denote the plus construction. Applying functorial factorization, we can arrange it so that the natural map $Y \rightarrow Y^{+}$is a cofibration.

If $(Y, h)$ is an object of $C_{E}$, then the 1-connectedness of $E$ implies $Y$ has trivial first homology. Therefore, $Y^{+}$is a 1 -connected space. So if $(Y, h) \rightarrow\left(Z, h^{\prime}\right)$ is a morphism, the induced map of plus constructions $Y^{+} \rightarrow Z^{+}$is a weak equivalence by Whitehead's theorem.

Since $E$ is fibrant and cofibrant, the space $\Omega^{\infty} E$ is cofibrant and 1-connected. The natural map $\Omega^{\infty} E \rightarrow\left(\Omega^{\infty} E\right)^{+}$is a cofibration which is a weak equivalence. Consequently, there is a retraction $r:\left(\Omega^{\infty} E\right)^{+} \rightarrow \Omega^{\infty} E$. 
Let $i: C_{E}^{\prime} \rightarrow C_{E}$ denote the inclusion. Define a functor

$$
\pi: C_{E} \rightarrow C_{E}^{\prime}
$$

by the rule $\pi(Y, h)=\left(Y^{+}, h^{b}\right)$, where $h^{b}: \Sigma^{\infty}\left(Y^{+}\right) \rightarrow E$ is adjoint to the composite

$$
Y^{+} \stackrel{(\hat{h})^{+}}{\rightarrow}\left(\Omega^{\infty} E\right)^{+} \stackrel{r}{\rightarrow} \Omega^{\infty} E
$$

Then there is an evident natural transformation from the identity functor of $C_{E}$ to the composite functor $i \circ \pi$. There is a similar evident natural transformation from the identity functor of $C_{E}^{\prime}$ to $\pi \circ i$. It follows that $i$ induces a homotopy equivalence on realizations.

\section{Proof of Theorem $\mathrm{A}$}

After defining the obstruction $\delta_{E}$, the idea of the remainder of the proof will be to construct a highly connected map from a suspension spectrum to $E$. The proof is then completed by applying Lemma 2.1

Definition of the obstruction. We describe below a certain fibration sequence of spectra

$$
D_{2}(E) \rightarrow S_{2}(E) \rightarrow E
$$

in which $S_{2}: \mathbf{S p} \rightarrow \mathbf{S p}$ is a certain homotopy functor.

Assuming this construction has been specified, we have:

Definition 3.1. The class

$$
\delta_{E} \in\left[E, \Sigma D_{2}(E)\right]
$$

is the obstruction to splitting the above fibration sequence, i.e., the homotopy class of its connecting map to the right.

Construction of the fibration. Consider the homotopy functor

$$
\Sigma^{\infty} \Omega^{\infty}: \mathbf{S p} \rightarrow \mathbf{S p}
$$

which assigns to a spectrum the suspension spectrum of its zero space. Let $S_{k}(E)$ denote the $k$-th stage of the Goodwillie tower of this functor and let $F_{k}(E):=$ fiber $\left(S_{k}(E) \rightarrow S_{k-1}(E)\right)$ denote the $k$-th layer.

The following result has been noted by several people, including Goodwillie, Arone, McCarthy, and Ahearn and Kuhn [A-K, Cor. 1.3].

Lemma 3.2. Assume $E$ is r-connected. Then the map

$$
\Sigma^{\infty} \Omega^{\infty} E \rightarrow S_{k}(E)
$$

is $((k+1) r+k+1)$-connected. Consequently, the Goodwillie tower of $\Sigma^{\infty} \Omega^{\infty} E$ is convergent if $E$ is 0-connected. Furthermore, there is a natural weak equivalence of functors

$$
F_{k}(E) \simeq D_{k}(E)
$$

Applying 3.2, we see that the bottom of the tower yields a fibration sequence of spectra

$$
D_{2}(E) \rightarrow S_{2}(E) \rightarrow E
$$

together with a $(3 r+3)$-connected map $\Sigma^{\infty} \Omega^{\infty} E \rightarrow S_{2}(E)$.

Corollary 3.3. Assume $E$ is $r$-connected and $\operatorname{dim} E \leq 3 r+3$. Then $\delta_{E}=0$ if and only if the map $\Sigma^{\infty} \Omega^{\infty} E \rightarrow E$ admits a section up to homotopy. 
Proof. The class $\delta_{E}$ is trivial if and only if the map $S_{2}(E) \rightarrow E$ has a section. The dimension constraint on $E$ and the lemma show that $S_{2}(E) \rightarrow E$ admits a section if and only if $\Sigma^{\infty} \Omega^{\infty} E \rightarrow E$ admits a section up to homotopy.

Now assume $E$ is $r$-connected, $r \geq 1, \operatorname{dim} E \leq 3 r+3$ and $\delta_{E}=0$. By the above remarks, we are entitled to choose a homotopy section $\sigma: E \rightarrow \Sigma^{\infty} \Omega^{\infty} E$. Let

$$
\Omega^{\infty} \sigma: \Omega^{\infty} E \rightarrow \Omega^{\infty} \Sigma^{\infty} \Omega^{\infty} E
$$

be the corresponding map of zero spaces.

There is another map $c: \Omega^{\infty} E \rightarrow \Omega^{\infty} \Sigma^{\infty} \Omega^{\infty} E$ (not an infinite loop map in general) which is defined by taking the adjoint to the identity map of $\Sigma^{\infty} \Omega^{\infty} E$.

Let $Y$ be the homotopy pullback of the maps $\Omega^{\infty} \sigma$ and $c$. Thus we have an $\infty$-cartesian square of spaces

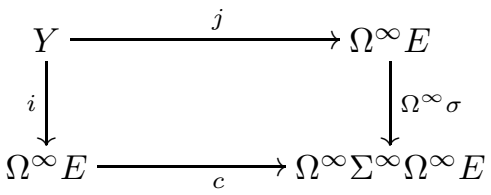

(commutative up to preferred homotopy).

Lemma 3.4. With respect to the above assumptions, let $\hat{j}: \Sigma^{\infty} Y \rightarrow E$ be the adjoint to the map labeled $j$ in diagram (2). Then $\hat{j}$ is $(3 r+2)$-connected.

Proof. Consider the diagram of spectra

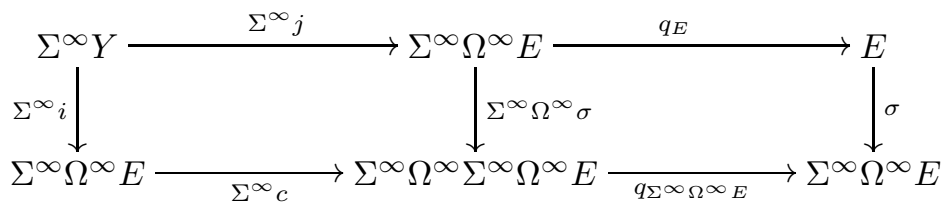

in which the maps labeled with $q$ are the counits to the adjunctions for $E$ and $\Sigma^{\infty} \Omega^{\infty} E$. The map $\hat{j}$ is therefore given by the composite $q_{E} \circ \Sigma^{\infty} j$, and the composite along the bottom, $q_{\Sigma^{\infty} \Omega^{\infty} E} \circ \Sigma^{\infty} c$, is clearly the identity. The left square commutes up to a preferred homotopy. The right square commutes on the nose.

The maps $\Omega^{\infty} \sigma$ and $c$ in diagram (2) are both $(2 r+1)$-connected. Consequently, by the dual Blakers-Massey theorem (see Go2]) diagram (2) is $(4 r+3)$-cocartesian. In particular, the left square in diagram (3) is a $(4 r+3)$-cocartesian square of spectra.

Regarding the right square in diagram (3), if $C$ denotes the homotopy cofiber of $\sigma$, then the evident map $C \rightarrow D_{2}(E)$ is $(3 r+3)$-connected. Similarly, if $C^{\prime}$ denotes the homotopy cofiber of $\Sigma^{\infty} \Omega^{\infty} \sigma$, an argument using the Blakers-Massey theorem, which we omit, shows that the evident map $C^{\prime} \rightarrow \Sigma^{\infty} \Omega^{\infty} D_{2}(E)$ is also $(3 r+3)$-connected.

Corollary [2.2] shows that the map $\Sigma^{\infty} \Omega^{\infty} D_{2}(E) \rightarrow D_{2}(E)$ is $(4 r+4)$-connected (because $D_{2}(E)$ is $(2 r+1)$-connected). We infer that the map $C^{\prime} \rightarrow C$ is also $(3 r+3)$-connected. Therefore, the right square in diagram (3) is $(3 r+3)$-cocartesian.

Putting both squares together, it follows that diagram (3) is $(3 r+3)$-cocartesian. Since the bottom composite is the identity map, we infer that the top composite $\hat{j}$ is $(3 r+2)$-connected, as asserted. 
Completion of the proof. Assume in addition to the above that $\operatorname{dim} E \leq 3 r+2$. Since the map $\hat{j}: \Sigma^{\infty} Y \rightarrow E$ is $(3 r+2)$-connected, we can apply Lemma 2.1, This gives a based space $Z$ and a based map $Z \rightarrow Y$ such that the composite $\Sigma^{\infty} Z \rightarrow$ $\Sigma^{\infty} Y \rightarrow E$ is a weak equivalence. The proof of Theorem $\mathrm{A}$ is now complete.

\section{Proof of Theorem B}

Step 1. Let $\mathcal{S}_{E}$ denote the set of homotopy classes of sections of the fibration $S_{2}(E) \rightarrow E$. Note that the abelian group $\left[E, D_{2}(E)\right]$ acts freely and transitively on $\mathcal{S}_{E}$ (cf. Lemma 6.1 below). Thus if $\mathcal{S}_{E}$ is given a basepoint, it follows that $\left[E, D_{2}(E)\right]$ and $\mathcal{S}_{E}$ are isomorphic (the isomorphism is dependent on the choice of basepoint).

There is a function

$$
\phi: \Theta_{E} \rightarrow \mathcal{S}_{E}
$$

defined by sending $(Y, h)$ to the class represented by

$$
E \simeq \Sigma^{\infty} Y \rightarrow S_{2}\left(\Sigma^{\infty} Y\right) \simeq S_{2}(E) .
$$

(The map in the middle is the preferred section of $S_{2}\left(\Sigma^{\infty} Y\right) \rightarrow \Sigma^{\infty} Y$, given by composing the map $\Sigma^{\infty} \Omega^{\infty}\left(\Sigma^{\infty} Y\right) \rightarrow S_{2}\left(\Sigma^{\infty} Y\right)$ with the map $\Sigma^{\infty} u: \Sigma^{\infty} Y \rightarrow$ $\Sigma^{\infty} \Omega^{\infty}\left(\Sigma^{\infty} Y\right)$, where $u: Y \rightarrow \Omega^{\infty} \Sigma^{\infty} Y$ is the adjoint of the identity map of $\Sigma^{\infty} Y$.)

If $E \rightarrow S_{2}(E)$ is a representative of $\mathcal{S}_{E}$, and $\operatorname{dim} E \leq 3 r+2$, we can perform the constructions in the previous section to get an element of $\Theta_{E}$ (the element is not necessarily unique). It is straightforward to check that this element of $\Theta_{E}$ maps to the given element of $\mathcal{S}_{E}$. Thus, when $\operatorname{dim} E \leq 3 r+2$, the function $\phi: \Theta_{E} \rightarrow \mathcal{S}_{E}$ is onto.

To complete the proof of Theorem B, it will be sufficient to show that $\phi$ is oneto-one when $\operatorname{dim} E \leq 3 r+1$. Choose a basepoint $(Y, h)$ for $\Theta_{E}$. Then $\mathcal{S}_{E}$ inherits a basepoint and $\mathcal{S}_{E}$ becomes identified with $\left[E, D_{2}(E)\right]$. Thus we may rewrite $\phi$ as a basepoint preserving function

$$
\Theta_{E} \rightarrow\left[E, D_{2}(E)\right]
$$

where the basepoint of the codomain is the zero element. Since $Y$ was chosen arbitrarily, it suffices to show that $\phi$ is one-to-one at the inverse image of the basepoint.

Step 2. We digress to develop a relative version of Theorem $\mathrm{A}$ Suppose that $A \longmapsto E$ is a cofibration in the category of spectra. We write $\operatorname{dim}(E, A) \leq n$ if $E$ is obtained from $A$ up to homotopy by attaching cells of dimension $\leq n$. Assume that $A=\Sigma^{\infty} Z$ is a suspension spectrum.

Theorem 4.1. There is an obstruction

$$
\delta_{(E, A)} \in\left[E / A, \Sigma D_{2}(E)\right]
$$

whose triviality is necessary to finding a cofibration $Z \rightarrow W$ and a weak equivalence $\Sigma^{\infty} W \simeq E$ extending the identity on $\Sigma^{\infty} Z=A$. If $E$ is $r$-connected $(r \geq 1)$ and $\operatorname{dim}(E, A) \leq 3 r+2$, then the vanishing of this obstruction is also sufficient.

The obstruction $\delta_{(E, A)}$ is defined in the same way as $\delta_{E}$ taking care to notice that the restriction of $\delta_{E}$ to $A$ has a preferred trivialization. The proof of Theorem 4.1 is virtually the same as the proof of Theorem $\mathrm{A}$ so we omit it. 
Step 3. We now return to the proof of Theorem B We shall apply Theorem 4.1 in the following situation: choose a representative $(Y, h)$ for an element in $\Theta_{E}$ (call this the basepoint). Suppose that $\left(Y^{\prime}, h^{\prime}\right)$ represents an another element in $\Theta_{E}$. Both elements combine to give a weak equivalence

$$
\Sigma^{\infty}\left(Y \vee Y^{\prime}\right) \stackrel{\sim}{\rightarrow} E \vee E .
$$

Applying Theorem 4.1 to the pair $\left(E \wedge I_{+}, E \vee E\right)$ we get a necessary obstruction

$$
\delta_{\left(E \wedge I_{+}, E \vee E\right)} \in\left[\Sigma E, \Sigma D_{2}(E)\right] \cong\left[E, D_{2}(E)\right]
$$

to finding a cofibration $Y \vee Y^{\prime} \longmapsto W$ of based spaces and a weak equivalence

$$
\left(\Sigma^{\infty} W, \Sigma^{\infty}\left(Y \vee Y^{\prime}\right)\right) \simeq\left(E \wedge I_{+}, E \vee E\right) .
$$

It follows from this that the zig-zag $Y \rightarrow W \leftarrow Y^{\prime}$ equates $(Y, h)$ with $\left(Y^{\prime}, h^{\prime}\right)$ in $\Theta_{E}$.

Thus, fixing $(Y, h)$ and allowing $\left(Y^{\prime}, h^{\prime}\right)$ to vary defines a function

$$
\Theta_{E} \rightarrow\left[E, D_{2}(E)\right]
$$

which is just another description of the function $\phi$ (we omit the details).

Assume that $E$ is $r$-connected and $\operatorname{dim} E \leq 3 r+1$. Then Theorem 4.1 shows that $\left(Y^{\prime}, h^{\prime}\right)$ maps to zero under $\phi$ if and only if $\left(Y^{\prime}, h^{\prime}\right)$ is the basepoint of $\Theta_{E}$. This completes the proof of Theorem $\mathrm{B}$.

\section{5. $\mathfrak{M}_{E}$ AS A CLASSIFYING SPACE}

Fix a cofibrant based space $Z$. Let $\mathbf{T o p}_{* / Z}$ denote the category of based spaces over $Z$. An object $y$ of this category consists of a based space $Y$ together with a based map $p_{Y}: Y \rightarrow Z$. A morphism $\left(Y, p_{Y}\right) \rightarrow\left(Y^{\prime}, p_{Y^{\prime}}\right)$ is given by a map of based spaces $f: Y \rightarrow Y^{\prime}$ such that $p_{Y^{\prime}} \circ f=p_{Y}$.

Since $\mathbf{T o p}_{* / Z}$ is an over category of the Quillen model category Top $_{*}$, it follows that it too has the structure of a Quillen model category (see $[\mathrm{Qu}]$ ). A weak equivalence is a morphism $y \rightarrow y^{\prime}$ whose underlying map of spaces is a weak homotopy equivalence. We say $y \rightarrow y^{\prime}$ is a fibration if its underlying map of spaces is. We say that $y \rightarrow y^{\prime}$ is a cofibration if it satisfies the left lifting property with respect to the acyclic fibrations.

Let $w \mathbf{T o p}_{* / Z}$ denote the subcategory consisting of the weak equivalences. Let $w_{(y)} \mathbf{T o p}_{* / Z}$ denote the full subcategory of $w \mathbf{T o p}{ }_{* / Z}$ consisting of those objects connected to $y$ by a chain of weak equivalences.

The proof of the following proposition, which we attribute to Waldhausen, is proved by the same method as Wa 2.2 .5$]$. We omit the details.

Proposition 5.1. Assume that $y$ is fibrant and cofibrant. Then there is a weak equivalence of spaces

$$
\left|w_{(y)} \mathbf{T o p}_{* / Z}\right| \simeq B G(y),
$$

where $G(y)$ denotes the topological monoid of self-homotopy equivalences of $y$ in Top $_{* / Z}$, and BG(y) is its classifying space.

We apply this in the following special case: let $E$ be a 1-connected fibrant and cofibrant spectrum. The category $C_{E}^{\prime}$, whose realization is the moduli space $\mathfrak{M}_{E}^{\prime}$ (cf. $\S 2$ ), is just the full subcategory of $w$ Top $_{* / \Omega^{\infty} E}$ whose objects $Y$ are such that the map $p_{Y}: Y \rightarrow \Omega^{\infty} E$ is adjoint to a weak equivalence of spectra. 
If we combine Proposition 5.1] with Proposition 2.3. we obtain

Corollary 5.2. Let $y$ be an object of $C_{E}$ which is fibrant and cofibrant when considered as an object of $\mathbf{T o p}_{* / \Omega^{\infty} E}$. Let $\mathfrak{M}_{E,(y)}$ be the connected component of $\mathfrak{M}_{E}$ which contains $y$. Then there is a homotopy equivalence

$$
\mathfrak{M}_{E,(y)} \simeq B G(y) .
$$

\section{Proof of Theorem C}

Outline of the proof. The basepoint $y=(Y, h)$ of $\mathfrak{M}_{E}$ can be taken as an object of $C_{E}$. Taking the plus construction if necessary, we can assume, without loss of generality, that $Y$ is 1-connected (see the argument in the proof of Proposition 2.3). We may also assume that $y$ is fibrant and cofibrant when considered as an object of $\mathbf{T o p}_{* / \Omega^{\infty} E}$.

We will construct a tower of fibrations of based spaces

$$
\cdots \rightarrow T_{3}(y) \rightarrow T_{2}(y) \rightarrow T_{1}(y)
$$

such that

- $T_{1}(y)$ is contractible;

- for $k>1$, there is a weak equivalence

$$
\operatorname{fiber}\left(T_{k}(y) \rightarrow T_{k-1}(y)\right) \simeq \Omega^{\infty}\left(W_{k} \wedge_{h \Sigma_{k}} Y^{\wedge k}\right),
$$

where $W_{k}$ denotes the spectrum that classifies the $k$-th layer of the Goodwillie tower of the identity functor on based spaces.

- there is a weak equivalence

$$
\Omega \mathfrak{M}_{E,(y)} \simeq \lim _{k} T_{y}(y) .
$$

Assuming this has been done, we can define the spectral sequence $\left\{E_{p, q}^{r}\right\}$ as the homotopy spectral sequence of the tower $\left\{T_{k}(y)\right\}_{k}$.

We now digress to discuss generalities about section spaces and the basic properties of the Goodwillie tower of the identity functor.

Digression. Suppose $p: E \rightarrow Z$ is a fibration of based spaces. We say that $p$ is principal if there exists a commutative $\infty$-cartesian square of based spaces

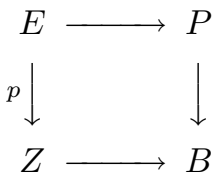

such that $P$ is contractible.

Suppose that $Z$ is connected. If $p: E \rightarrow Z$ is principal, there is an "action" $\Omega B \times E \rightarrow E$. If there is a section $Z \rightarrow E$ one can combine it with this action to produce a map of fibrations $\Omega B \times Z \rightarrow E$ covering the identity map of $Z$. This implies that $p$ is weak fiber homotopically trivial. Let $\operatorname{Sec}(p)$ denote the space of sections of $p$. Then we have shown

Lemma 6.1. Assume $p: E \rightarrow Z$ is principal. Assume that $\operatorname{Sec}(p)$ is nonempty and comes equipped with basepoint. Then there is a weak equivalence of based spaces

$$
\operatorname{Sec}(p) \simeq F(Z, \Omega B) .
$$


We next recall for the reader the basic properties of the Goodwillie tower of the identity functor on based spaces (cf. Goodwillie [Go1], Go2], Go3], Johnson [Jo], Arone $[\mathrm{Ar}]$ ).

Theorem 6.2. There is a tower of fibrations of homotopy functors on based spaces

$$
\cdots \rightarrow P_{2}(X) \rightarrow P_{1}(X)
$$

and compatible natural transformations $X \rightarrow P_{k}(X)$ such that

- $P_{1}(X)=Q(X)$ is the stable homotopy functor;

- For $k \geq 2$, the fibration $P_{k}(X) \rightarrow P_{k-1}(X)$ is principal;

- the $k$-the layer $L_{k}(X)$ is naturally weak equivalent to the functor

$$
X \mapsto \Omega^{\infty}\left(W_{k} \wedge_{h \Sigma_{k}} X^{\wedge k}\right) ;
$$

- if $X$ is 1-connected, then the natural map

$$
X \rightarrow \lim _{k} P_{k}(X)
$$

is a weak equivalence.

Completion of the proof. We are now in a position to define the tower $\left\{T_{k}(y)\right\}_{k}$.

Definition 6.3. Let $T_{k}(y)$ be the space of lifts

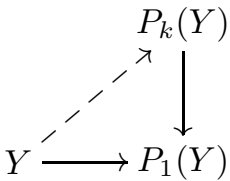

where $Y \rightarrow P_{1}(Y)$ is the natural map. Note that $T_{k}(y)$ comes equipped with a basepoint defined by the natural map $Y \rightarrow P_{k}(Y)$.

From the definition of $T_{k}(y)$, there is an evident tower of fibrations of based spaces

$$
\cdots \rightarrow T_{2}(y) \rightarrow T_{1}(y)
$$

with $T_{1}(y)=*$. Furthermore, the fiber of the map $T_{k}(y) \rightarrow T_{k-1}(y)$ at the basepoint is identified with the space of lifts

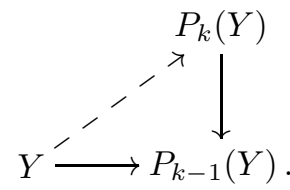

Note that this is just the space of sections of the pulled back fibration $Y \times_{P_{k-1}(Y)}$ $P_{k}(Y) \rightarrow Y$. The latter fibration is principal and comes equipped with a preferred section.

Applying Lemma 6.1 and Theorem 6.2 we obtain a weak equivalence of based spaces

$$
\operatorname{fiber}\left(T_{k}(y) \rightarrow T_{k-1}(y)\right) \simeq F\left(Y, \Omega^{\infty}\left(W_{k} \wedge_{h \Sigma_{k}} Y^{\wedge k}\right)\right) .
$$

Taking adjunctions, we see that $F\left(Y, \Omega^{\infty}\left(W_{k} \wedge_{h \Sigma_{k}} Y^{\wedge k}\right)\right)$ is weak equivalent to the space $F\left(E, W_{k} \wedge_{h \Sigma_{k}} E^{\wedge k}\right)$ (where we are using the identification $\Sigma^{\infty} Y \simeq E$ ). 
To complete the proof of Theorem $\mathrm{C}$ it suffices to identify the inverse limit of the tower $\left\{T_{k}(y)\right\}_{k}$. It is clear that this inverse limit is just the space of lifts

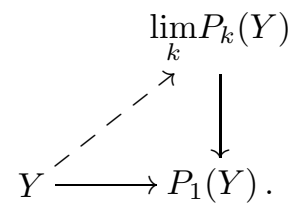

Recall that $P_{1}(Y)=Q(Y)$ and since $Y$ is 1-connected, $\lim _{k} P_{k}(Y) \simeq Y$. With respect to these identifications, we infer that this space of lifts is weak equivalent to the underlying space of the topological monoid $G(y)$. Applying Corollary 5.2 and Proposition 2.3 completes the proof of Theorem [C]

\section{Proof of Theorem D}

Let $\operatorname{tr}: D_{2}(E) \rightarrow E^{\wedge 2}$ be the transfer and let $\pi: E^{\wedge 2} \rightarrow D_{2}(E)$ be the projection ([L-M-S, Chap. 4]). It is well known that the composite

$$
p \circ \operatorname{tr}: D_{2}(E) \rightarrow D_{2}(E)
$$

is a weak equivalence after inverting 2 . To prove the first part of Theorem $\mathrm{D}$, it is clearly enough to show

Lemma 7.1. For a connected spectrum E, the class

$$
(\Sigma \operatorname{tr}) \circ \delta_{E} \in[E, \Sigma E \wedge E]
$$

is trivial.

Proof. This is a consequence of a result of Ahearn and Kuhn (see [A-K, Cor. 1.7] in conjunction with the discussion after $[\mathrm{A}-\mathrm{K}$. Cor. 1.2]). For an alternative proof, see the remark after Corollary 10.5 below.

In the case we need, Ahearn and Kuhn construct a natural transformation $\Phi: S_{2}(E) \rightarrow E \wedge E$ fitting into a preferred homotopy commutative diagram

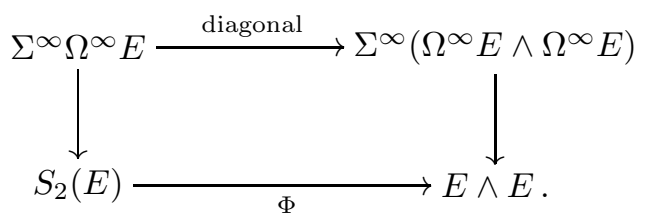

They then identify the composite map

$$
D_{2}(E) \rightarrow S_{2}(E) \stackrel{\Phi}{\rightarrow} E \wedge E
$$

with the transfer ${ }^{2}$

Since $E \mapsto E^{\wedge 2}$ is a homogeneous quadratic functor, it coincides with the second stage of its Goodwillie tower and its first stage is trivial. So the map of Goodwillie

\footnotetext{
${ }^{2}$ Randy McCarthy has informed me that that this result is also implicit in the recent Ph.D. thesis of K.B. Bauer $\mathrm{Ba}$.
} 
towers associated with $\Phi$ in degrees $\leq 2$ may be displayed as

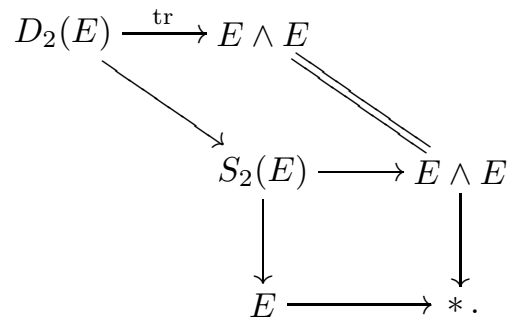

If we take horizontal homotopy cofibers of the maps from stage two to stage one, we obtain a homotopy commutative diagram

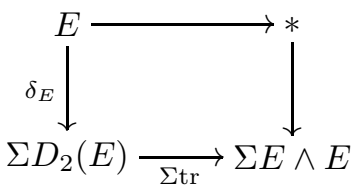

which shows that $(\Sigma \operatorname{tr}) \circ \delta_{E}$ is null homotopic.

We now prove the second part of Theorem $\mathrm{D}$. Let $f: E \rightarrow E^{\prime}$ be a map which is a 2-local weak equivalence. Assume that $E$ and $E^{\prime}$ satisfy the connectivity hypotheses of Theorem B. Then the naturality of the obstruction shows that the diagram

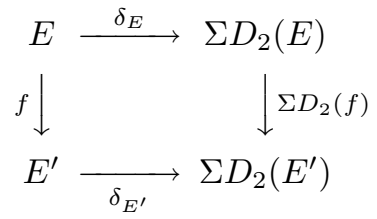

is homotopy commutative. If we invert 2 , then by the first part, the obstructions $\delta_{E}$ and $\delta_{E}^{\prime}$ vanish. It we localize at 2 , then $f$ and $\Sigma D_{2}(f)$ become weak equivalences, and therefore $\delta_{E}$ and $\delta_{E^{\prime}}$ coincide. Thus, integrally, $\delta_{E}=0$ if and only $\delta_{E}^{\prime}=0$.

\section{Illustrations of Theorem B}

We give two examples.

Two cells. For $p>1$ and $q \leq 3 p-3$, consider the suspension spectrum $E=$ $\Sigma^{\infty}\left(S^{p} \cup_{f} e^{q+1}\right)$ where $f: S^{q} \rightarrow S^{p}$ is some map. According to Theorem $\mathrm{B}$ we have

$$
\Theta_{E} \cong\left[E, D_{2}(E)\right] \cong \pi_{q+1}\left(D_{2}\left(S^{p}\right)\right),
$$

where the second of these isomorphisms comes from that fact that a homotopy class $E \rightarrow D_{2}(E)$ maps the top cell of $E$ into $D_{2}\left(S^{p}\right) \subset D_{2}(E)$.

The first nontrivial group occurs when $q=2 p-1$. The group $\pi_{2 p}\left(D_{2}\left(S^{p}\right)\right)$ is isomorphic to $\mathbb{Z}$ if $p$ is even and $\mathbb{Z}_{2}$ if $p$ is odd. The distinct elements of $\Theta_{E}$ are represented by the suspension spectra of $S^{p} \cup_{g} e^{2 p}$, with

$$
g=f+k[\iota, \iota],
$$

where $k$ is an integer $(\bmod 2$ if $p$ is odd), and $[\iota, \iota]$ is the Whitehead product. The group structure on $\Theta_{E}$ is given by adding these integers. 
In fact, Mahowald has computed $\pi_{q+1}\left(D_{2}\left(S^{p}\right)\right)$ for $q \leq \min (3 p-3,2 p+29)$, at the prime 2 (see [Mah, Table 4.1], see also Milgram [Mi, Table 13.5]). For example, assuming in addition $p \equiv 1 \bmod 16$, the first few groups are:

\begin{tabular}{|c|c|c|c|c|c|c|c|}
\hline$j$ & 0 & 1 & 2 & 3 & 4 & 5 & 6 \\
\hline$\pi_{2 p+j}\left(D_{2}\left(S^{p}\right)\right)$ & $\mathbb{Z}_{2}$ & $\mathbb{Z}_{2}$ & $\mathbb{Z}_{8}$ & $\mathbb{Z}_{2}$ & 0 & $\mathbb{Z}_{2}$ & $\mathbb{Z}_{16} \oplus \mathbb{Z}_{2}$ \\
\hline
\end{tabular}

Tori. Let $E=\Sigma^{\infty}\left(S^{p} \times S^{p}\right)$, with $p>1$. Then

$$
\Theta_{E} \cong\left[E, D_{2}(E)\right] \cong \pi_{2 p}\left(D_{2}\left(S^{p} \vee S^{p}\right)\right) .
$$

When $p$ is even, the group $\pi_{2 p}\left(D_{2}\left(S^{p} \vee S^{p}\right)\right)$ is isomorphic to $\mathbb{Z}^{\oplus 3}$. When $p$ is odd, it is isomorphic to $\mathbb{Z}_{2} \oplus \mathbb{Z}_{2} \oplus \mathbb{Z}$. The elements of $\Theta_{E}$ can be represented by the suspension spectra of the complexes

$$
\left(S^{p} \vee S^{p}\right) \cup_{h} e^{2 p}
$$

with attaching map

$$
h=k x \circ[\iota, \iota]+\ell y \circ[\iota, \iota]+m \omega,
$$

where $\omega$ is the attaching map for the top cell of $S^{p} \times S^{p}, x$ and $y$ are the summand inclusions $S^{p} \rightarrow S^{p} \vee S^{p}$, and $k, \ell$ and $m$ are integers (take $k$ and $\ell$ modulo 2 if $p$ is odd). Hence the elements of $\Theta_{E}$ are specified by triples $(k, \ell, m)$. The identity element of $\Theta_{E}$ is $(0,0,1)$ and addition is given by

$$
(k, \ell, m)+\left(k^{\prime}, \ell^{\prime}, m^{\prime}\right)=\left(k+k^{\prime}, \ell+\ell^{\prime}, m+m^{\prime}-1\right) .
$$

\section{LOOSE ENDS}

Relation to the James-Hopf invariant. Another approach to desuspension questions is to inductively desuspend cell-by-cell (with respect to a cell decomposition of $E$ ). We explain here how this relates to our approach.

The idea is this: when $E$ can be written as a homotopy cofiber of a map of suspension spectra $f: \Sigma^{\infty} A \rightarrow \Sigma^{\infty} B$, it turns out that $\delta_{E}$ is closely related to the James-Hopf invariant

$$
H_{2}(f) \in\left[\Sigma^{\infty} A, D_{2}\left(\Sigma^{\infty} B\right)\right] .
$$

Explicitly, there is a homomorphism

$$
\psi:\left[\Sigma^{\infty} A, D_{2}\left(\Sigma^{\infty} B\right)\right] \rightarrow\left[E, \Sigma D_{2}(E)\right]
$$

given by suspending, precomposing with the connecting map $E \rightarrow \Sigma \Sigma^{\infty} A$ and postcomposing with the inclusion $\Sigma D_{2}\left(\Sigma^{\infty} B\right) \rightarrow \Sigma D_{2}(E)$. We assert that $\psi$ maps $H_{2}(f)$ to $\delta_{E}$ (we defer the proof to another paper).

If we also assume that $B$ is $r$-connected $(r \geq 1), \operatorname{dim} B \leq 2 r+1, A$ is $2 r$ connected and $\operatorname{dim} A \leq 3 r+1$, then we infer that $E$ is $r$-connected and $\operatorname{dim} E \leq$ $3 r+2$. Furthermore, obstruction theory implies that $\psi$ is an isomorphism. We conclude that $E$ desuspends if and only if $H_{2}(f)=0$ (the "if" part of this statement is well known). 
Musings on the spectral sequence. It would be desirable to have a version of the spectral sequence in Theorem $\mathbb{C}$ which converges to $\pi_{*}\left(\mathfrak{M}_{E}\right)$ in degree zero.

The spectral sequence still lacks a geometric interpretation. Conjecturally, the spectral sequence should be a packaging machine for the obstructions to equipping a spectrum with the structure of an

" $E_{\infty}$-coalgebra over the sphere spectrum".

What I have in mind here comes from the observation that for finite spectra $E$, one can show that $S_{2}(E)$ is a model for $(E \wedge E)^{\mathbb{Z}_{2}}=$ the categorical fixed points of $\mathbb{Z}_{2}$ acting on $E \wedge E$, and a choice of section for the fibration $S_{2}(E) \rightarrow E$ can be thought of as commutative (but not necessarily associative) "diagonal" for $E$.

It is tempting to conjecture that the spectral sequence arises from a tower whose $k$-stage encodes, in some sense, the moduli space of spectra equipped with the choice of commutative diagonal that is coherently homotopy associative up to order $k-1$.

\section{Appendix: On quadratic homotopy functors}

The invariant $\delta_{E}$ was defined as the connecting map associated with the degree two homotopy functor $E \mapsto S_{2}(E)$. It is not difficult to classify the quadratic homotopy functors from spectra to spectra. The classification, which is expressed in terms of the Tate construction, gives a more concrete way of understanding $\delta_{E}$. I would like to thank Tom Goodwillie and Nick Kuhn for explaining these ideas to me.

Let $f: \mathbf{S p} \rightarrow \mathbf{S p}$ be a homotopy functor of degree $\leq 2$. This means that $f$ maps strongly cocartesian 3 -cubes to $\infty$-cartesian $(=\infty$-cocartesian $) 3$-cubes. We also require $f$ to be stably excisive (see [Go2] for the meaning of this language). We also assume that $f$ is reduced in the sense that its value at the zero object is contractible.

If we additionally assume that $f$ commutes with filtered homotopy colimits, then the general theory describes $f$ up to homotopy as sitting within a fibration sequence

$$
W_{2} \wedge_{h \mathbb{Z}_{2}} E^{\wedge 2} \rightarrow f(E) \rightarrow W_{1} \wedge E,
$$

where $W_{1}$ is a spectrum and $W_{2}$ is a spectrum with $\mathbb{Z}_{2}$-action (we remind the reader that $W_{2} \wedge_{h \mathbb{Z}_{2}} E^{\wedge 2}$ denotes the homotopy orbits of $\mathbb{Z}_{2}$ acting diagonally on $\left.W_{2} \wedge E^{\wedge 2}\right)$. The connecting map of this sequence is then a natural transformation

$$
u_{f}(E): W_{1} \wedge E \rightarrow \Sigma\left(W_{2} \wedge_{h \mathbb{Z}_{2}} E^{\wedge 2}\right) .
$$

Renaming $W:=W_{1}$ and $V:=\Sigma W_{2}$, we can rewrite $u_{f}(E)$ as

$$
W \wedge E \rightarrow V \wedge_{h \mathbb{Z}_{2}} E^{\wedge 2} .
$$

Notice that $u_{f}(E)$ is a natural transformation from the linear functor $E \mapsto W \wedge E$ to the homogeneous quadratic functor $E \mapsto V \wedge_{h \mathbb{Z}_{2}} E^{\wedge 2}$, and clearly, $f(E)$ is identified with the homotopy fiber of $u_{f}(E)$. We will show how to classify such natural transformations.

Let $\alpha$ denote the unique nontrivial one-dimensional real representation of $\mathbb{Z}_{2}$. If $j \geq 0$ is any integer, let $S^{j \alpha}$ be the suspension spectrum of the one point compactification of the direct sum of $j$-copies of $\alpha$. If $j<0$, define $S^{j \alpha}$ as $F\left(S^{-j \alpha}, S^{0}\right)$, the Spanier-Whitehead dual of $S^{-j \alpha}$. Note that $S^{j \alpha}$ has a $\mathbb{Z}_{2}$-action, and there is an evident cofibration sequence of spectra with $\mathbb{Z}_{2}$-action

$$
S^{(j-1) \alpha} \rightarrow S^{j \alpha} \rightarrow\left(\mathbb{Z}_{2}\right)_{+} \wedge S^{j}
$$


where the third term is given by inducing up the $j$-sphere with trivial action to a spectrum with free $\mathbb{Z}_{2}$-action.

Let us now consider $u_{f}$ applied to $S^{j} \wedge E$. Then we have

$$
u_{f}\left(S^{j} \wedge E\right): W \wedge E \wedge S^{j} \rightarrow\left(V \wedge E^{\wedge 2}\right) \wedge_{h \mathbb{Z}_{2}}\left(S^{j} \wedge S^{j \alpha}\right)
$$

where we have taken the liberty of rewriting $S^{j} \wedge S^{j}$ with tranposition action as $S^{j} \wedge S^{j \alpha}$. If we desuspend $j$-times, we get for each $j$ a map

$$
\Sigma^{-j} u_{f}\left(S^{j} \wedge E\right): W \wedge E \rightarrow\left(V \wedge E^{\wedge 2}\right) \wedge_{h \mathbb{Z}_{2}} S^{j \alpha}
$$

Lemma 10.1. These maps are compatible in the sense that the diagram

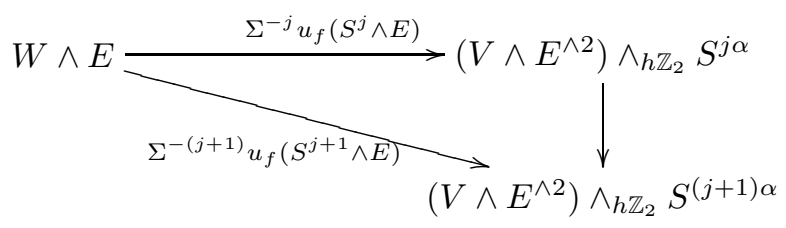

is homotopy commutative.

Proof. If we apply $u_{f}$ to the evident cocartesian square of spectra

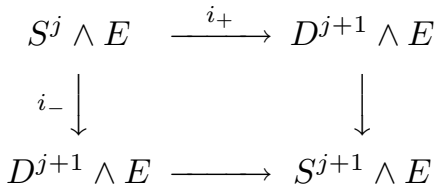

we obtain a map of squares of spectra. This map induces a homotopy commutative diagram

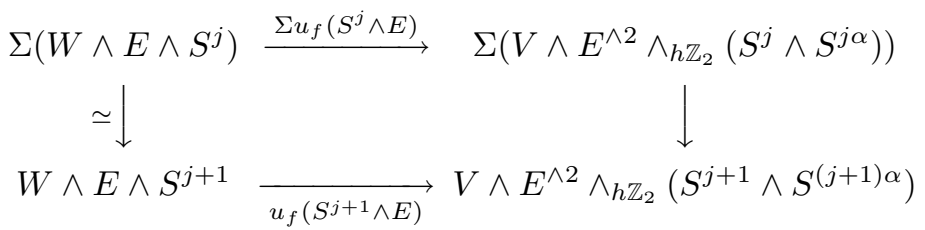

where the vertical maps are induced by maps from the pushouts coming from $i_{ \pm}$. The proof is now completed by desuspending $j+1$ times.

Recall that any spectrum $U$ with (naive) $\mathbb{Z}_{2}$-action comes equipped with a norm map $U_{h \mathbb{Z}_{2}} \rightarrow U^{h \mathbb{Z}_{2}}$ from homotopy orbits to homotopy fixed points (for details, see A-C-D], [W-W,$\S 2], \mathrm{G}-\mathrm{M}], \mathrm{K} 12])$. Recall that the Tate spectrum

$$
U^{t \mathbb{Z}_{2}}
$$

is the homotopy cofiber of the norm map. Thus we have a norm sequence

$$
U_{h \mathbb{Z}_{2}} \rightarrow U^{h \mathbb{Z}_{2}} \rightarrow U^{t \mathbb{Z}_{2}}
$$

The following result appears in the literature in several places (see, e.g., the beginning of the proof of theorem 16.1 in G-M]):

Lemma 10.2. There is a weak equivalence

$$
U^{t \mathbb{Z}_{2}} \simeq \Sigma\left(\operatorname{holim}_{j} U \wedge_{h \mathbb{Z}_{2}} S^{j \alpha}\right)
$$


Let

$$
u_{f}^{b}(E): W \wedge E \rightarrow \Sigma^{-1}\left(V \wedge E^{\wedge 2}\right)^{t \mathbb{Z}_{2}}
$$

be the natural transformation resulting from Lemmas 10.1 and 10.2. From the definition of $u_{f}^{b}(E)$, we conclude

Corollary 10.3 (Compare [Mc2, Prop. 4]). Up to homotopy, we have a factorization

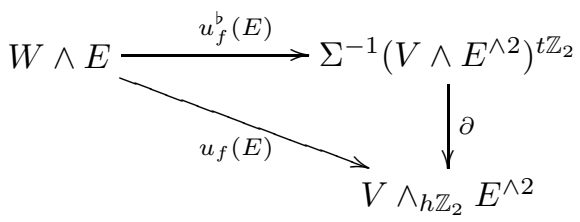

where $\partial$ is the connecting map in the norm sequence.

We next need to know that the target of $u_{f}^{b}(E)$ is a homology theory in $E$. This is the content of the next lemma (compare [J-W, Thm. 3.2] and [Mc2, Prop. 4]).

Lemma 10.4. The functor

$$
E \mapsto \Sigma^{-1}\left(V \wedge E^{\wedge 2}\right)^{t \mathbb{Z}_{2}}
$$

has degree $\leq 1$.

Proof. (Sketch) We will only give the proof when $E$ varies over the category of finite spectra. For the general case, see [Mc2] 3

There is a cofibration sequence

$$
\Sigma^{-1}\left(V \wedge E^{\wedge 2}\right)^{t \mathbb{Z}_{2}} \rightarrow V \wedge_{h \mathbb{Z}_{2}} E^{\wedge 2} \rightarrow\left(V \wedge E^{\wedge 2}\right)^{h \mathbb{Z}_{2}}
$$

All functors in this sequence have degree $\leq 2$. The functor in the middle is homogeneous of degree two, and hence, its linearization vanishes. A direct calculation which we omit shows that the homogeneous degree two part of $E \mapsto\left(V \wedge E^{\wedge 2}\right)^{h \mathbb{Z}_{2}}$ coincides with $E \mapsto V \wedge_{h \mathbb{Z}_{2}} E^{\wedge 2}$ (this is where the assumption that $E$ ranges over finite spectra is used, for the functor $E \mapsto\left(V \wedge E^{\wedge 2}\right)^{h \mathbb{Z}_{2}}$ doesn't necessarily preserve filtered homotopy colimits). We infer that $E \mapsto \Sigma^{-1}\left(V \wedge E^{\wedge 2}\right)^{t \mathbb{Z}_{2}}$ has trivial homogeneous degree two part; it therefore has degree $\leq 1$.

A consequence of this lemma is that the natural transformation $u_{f}^{b}$, when restricted to finite spectra, is determined by its value at $S^{0}$; i.e., for finite spectra $E$ we have $u_{f}^{b}(E) \simeq u_{f}^{b}\left(S^{0}\right) \wedge \operatorname{id}_{E}$. Since $u_{f}(E) \simeq \partial \circ u_{f}^{b}(E)$, where $\partial$ is independent of $f$, it follows that $u_{f}$ is determined by $u_{f}^{b}\left(S^{0}\right)$. As $f$ is determined by its restriction to finite spectra, we conclude

Corollary 10.5. The functor $f$ is determined up to weak equivalence by the homotopy class

$$
\left[u_{f}^{b}\left(S^{0}\right)\right] \in\left[W, \Sigma^{-1} V^{t \mathbb{Z}_{2}}\right] .
$$

Remark 10.6. The above analysis yields an alternative proof of Theorem $\mathrm{D}$. The point is that away from 2 , the connecting map $\partial$ in the norm sequence is null homotopic.

\footnotetext{
${ }^{3}$ I wish to thank the referee for pointing out that the lemma holds without the finiteness hypothesis.
} 
We now apply 10.5 in the case of the functor $E \mapsto S_{2}(E)$. Here, $W=S^{0}$ and $V=S^{1}$ (with trivial action). Furthermore, the natural transformation $u_{f}(E)$ is just $\delta_{E}$.

We see that $\delta_{E}$ is determined by a certain homotopy class

$$
\delta^{b} \in\left[S^{0},\left(S^{0}\right)^{t \mathbb{Z}_{2}}\right] .
$$

With slightly more care, it is not difficult to give an explicit construction: let $\iota: S^{0} \rightarrow\left(S^{0}\right)^{h \mathbb{Z}_{2}}$ be adjoint to the evident unit map $\left(B \mathbb{Z}_{2}\right)_{+} \rightarrow S^{0}$. Then $\delta^{b}$ is represented by the composite

$$
S^{0} \stackrel{\iota}{\rightarrow}\left(S^{0}\right)^{h \mathbb{Z}_{2}} \rightarrow\left(S^{0}\right)^{t \mathbb{Z}_{2}} .
$$

According to the Segal conjecture $[\mathrm{C}], \pi_{0}\left(\left(S^{0}\right)^{t \mathbb{Z}_{2}}\right)$ is isomorphic to the 2-adic integers. The class $\delta^{b}$ represents a topological generator of this group.

\section{ACKNOWLEDGEMENTS}

This paper would not have come into being had I not had the fortune of discussing mathematics with Greg Arone, Randy McCarthy and Tom Goodwillie in the middle to late 1990s. I am indebted to them for their help. I also owe much to Bill Richter, who explained to me the proof of the Berstein-Hilton theorem back in the 1980s.

I am grateful to Bob Bruner for explaining to me how to compute the stable homotopy of stunted projective space in low degrees, and for drawing my attention to the early work of Kuhn. I would like to thank Nick Kuhn for some very helpful remarks, especially concerning the proof of Lemma 7.1 .

\section{REFERENCES}

[A-C-D] Adem, A., Cohen, R.L., Dwyer, W.G.: Tate homology, homotopy fixed points and the transfer. In: Algebraic Topology (Evanston 1988) Contemp. Math. 96, pp. 1-13. AMS 1989 MR 90k:55012

[A-K] Ahearn, S. T., Kuhn, N. J.: Product and other fine structure in polynomial resolutions of mapping spaces. Algebr. Geom. Topol. 2, 1123-1150 (2002) MR 2003j:55009

[Ar] Arone, G.: A generalization of the Snaith-type filtration. Trans. Amer. Soc. 351, 591-647 (1999) MR 99i:55011

[B-H] Berstein, I., Hilton, P. J.: On suspensions and comultiplications. Topology 2, 73-82 (1963) MR 27:762

[Ba] Bauer, K. B.: On Hopf algebra type and rational calculus decompositions. Ph.D. thesis, University of Illinois Urbana-Champaign 2001

[C] Carlsson, G.: Equvariant stable homotopy and Segal's Burnside ring conjecture. Ann. of Math. 120, 189-224 (1984) MR 86f:57036

[Go1] Goodwillie, T. G.: Calculus I, the derivative of a homotopy functor. K-theory 4, 1-27 (1990) MR 92m:57027

[Go2] Goodwillie, T. G.: Calculus II: analytic functors. K-theory 5, 295-332 (1992) MR 93i:55015

[Go3] Goodwillie, T. G.: Calculus III: Taylor series. Geom. Topol. 7, 645-711 (2003).

[Gr] Gray, B.: Desuspension at an odd prime. In: Algebraic topology, Aarhus 1982, Springer LNM 1051, pp. 360-370. Springer, 1984 MR 86d:55017

[G-M] Greenlees, J., May, J.: Generalized Tate Cohomology. (AMS MDual calculus for functors to spectra. emoirs, Vol. 543). AMS 1995 MR 96e:55006

[Jo] Johnson, B.: The derivatives of homotopy theory. Trans. Amer. Math. Soc. 347, 12951321 (1995) MR 96b:55012

[J-W] Jones, J.D.S., Wegmann S.A.: Limits of stable homotopy and cohomotopy groups. Math. Proc. Camb. Phil. Soc. 94, 473-482 (1983) MR 85b:55014

[Kl1] Klein, J. R.: Poincaré embeddings and fiberwise homotopy theory. Topology 38, 597-620 (1999) MR 2000b:57037 
[Kl2] Klein, J. R.: Axioms for generalized Farrell-Tate cohomology. J. Pure Appl. Algebra 172(2-3), 225-238 (2002) MR 2003d:55006

$[\mathrm{Ku}] \quad$ Kuhn, N. J.: Suspension spectra and homology equivalences. Trans. Amer. Math. Soc. 283, 303-313 (1984). MR 85g:55014

[L-M-S] Lewis, L. G., May, J. P., Steinberger, M., McClure, J. E.: Equivariant Stable Homotopy Theory. (LNM, Vol. 1213). Springer, 1986 MR 88e:55002

[Mah] Mahowald, M.: The metastable homotopy of $S^{n}$. (Memoirs of the Amer. Math. Soc, No. 72). Amer. Math. Soc. 1967 MR 38:5216

[Mc1] McCarthy, R.: On n-excisive functors of module categories. to appear in Math. Proc. Camb. Phil. Soc.

[Mc2] McCarthy, R.: Dual calculus for functors to spectra. Homotopy methods in algebraic topology (Boulder, CO 1999). (Contemp. Math. 271), Amer. Math. Soc. 2001 MR 2002c: 18009

[Mi] Milgram, R. J.: Unstable Homotopy from the Stable Point of View. (LNM, Vol. 368), Springer, 1974 MR 50:1235

[Qu] Quillen, D.: Homotopical Algebra. (LNM, Vol. 43). Springer, 1967 MR 36:6480

[Sc] Schwede, S.: Spectra in model categories and applications to the algebraic cotangent complex. J. Pure Appl. Algebra 120, 77-104 (1997) MR 98h:55027

[Wa] Waldhausen, F.: Algebraic $K$-theory of spaces. In: Algebraic and geometric topology, Proceedings Rutgers 1983, LNM 1126, pp. 318-419. Springer, 1985 MR 86m:18011

[W-W] Weiss, M., Williams, B.: Automorphisms of manifolds and algebraic $K$-theory. II. J. Pure Appl. Algebra 62, 47-107 (1989) MR 91e:57055

Department of Mathematics, Wayne State University, Detroit, Michigan 48202

E-mail address: klein@math. wayne.edu 